\title{
Utilisation du bois dans les pêcheries côtières du
} Cameroun

Use of the wood in coastal fisheries zones of Cameroun

Hyacinthe Angoni, Alphonse Pouokam Tatchim, Bernard Aloys

Nkonmeneck et Elie Nguekam

\section{(2) OpenEdition}

12 Journals

Édition électronique

URL : http://journals.openedition.org/ethnoecologie/2166

DOI : 10.4000/ethnoecologie.2166

ISSN : 2267-2419

Éditeur

Laboratoire Eco-anthropologie et Ethnobiologie

Référence électronique

Hyacinthe Angoni, Alphonse Pouokam Tatchim, Bernard Aloys Nkonmeneck et Elie Nguekam, " Utilisation du bois dans les pêcheries côtières du Cameroun », Revue d'ethnoécologie [En ligne], 7| 2015, mis en ligne le 30 juin 2015, consulté le 19 avril 2019. URL : http://journals.openedition.org/ ethnoecologie/2166 ; DOI : 10.4000/ethnoecologie.2166

Ce document a été généré automatiquement le 19 avril 2019

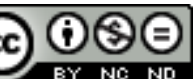

Revue d'ethnoécologie est mis à disposition selon les termes de la licence Creative Commons Attribution - Pas d'Utilisation Commerciale - Pas de Modification 4.0 International. 


\title{
Utilisation du bois dans les pêcheries côtières du Cameroun
}

Use of the wood in coastal fisheries zones of Cameroun

\author{
Hyacinthe Angoni, Alphonse Pouokam Tatchim, Bernard Aloys \\ Nkonmeneck et Elie Nguekam
}

\section{Introduction}

1 Au Cameroun les forêts de mangroves occupent une superficie d'environ 250000 hectares et comptent parmi les peuplements les plus étendus d'Afrique. Ces mangroves sont regroupées en trois grands ensembles. Dans l'estuaire du Rio del Rey (à la frontière avec le Nigéria), elles représentent $54 \%$ de la végétation, dans l'estuaire du Cameroun (autour de la ville de Douala) $45 \%$, et dans un ensemble de poches principalement autour de l'estuaire du Ntem (avec les fleuves Lokoundje, Nyong et Ntem) $1 \%$. Elles font partie du grand ensemble régional des mangroves du Golfe de Guinée (FAO 2006).

Dans les campements de pêche de la côte atlantique du Cameroun le long des côtes de la réserve de faune de Douala-Edéa et sur les côtes entre Kribi et Campo, le bois de mangrove est coupé à un rythme quotidien incessant pour construire des baraques en planche de palétuvier, des pirogues mais surtout pour en faire du combustible pour le fumage $\mathrm{du}$ poisson. Le fumage du poisson est une activité répandue en Afrique, principalement en Afrique de l'Ouest et en Afrique Centrale. Cette transformation du poisson a longtemps été un des seuls moyens de conservation, et a permis d'assurer une sécurité alimentaire. Les mangroves sont par excellence d'importantes zones de pêches ; elles fournissent sur le circuit commercial environ 60000 tonnes de poissons fumés par an (Mbog 2005). Les mangroves jouent deux rôles principaux sur l'écosystème côtier. Elles contribuent à la stabilisation de la ligne côtière et offrent d'importantes zones de frayères pour le développement des alevins. Le bois des mangroves a des propriétés calorifiques apprécié par les fumeurs de poissons. Les qualités organoleptiques du poisson fumé par le 
bois des mangroves sont aussi autant appréciées. Ainsi environ 60000 tonnes de poissons sont fumés par le bois de mangroves.

3 L'écosystème des mangroves joue à la fois le rôle de zone de reproduction pour les poissons et de provision de bois de feu, notamment le Rhizophora sp., recherché par les fumeuses à cause de son pouvoir calorifique et sa capacité à se consumer lentement, sans séchage préalable (Ajonina \& Usongo 2001). La surexploitation de la mangrove (Chesnes 2009) menace donc la pérennité de l'activité à deux niveaux : tout d'abord en menaçant l'approvisionnement en bois de feu à long terme, mais aussi en supprimant la niche de reproduction des ressources halieutiques, au risque de voir les stocks de poissons diminuer.

4 L'exploitation non rationalisée des zones côtières par les populations riveraines et par les divers opérateurs économiques (urbanisation anarchique, activités de tourisme et exploitation pétrolière) gagne du terrain et entraîne une dégradation de ces milieux avec diverses formes de pollution, par les déchets ménagers, les billes de bois abandonnées sur les plages, les hydrocarbures, les produits chimiques (Angoni 2014). La pression démographique et le développement de l'industrie touristique sur ces zones risquent d'entraîner une disparition de certaines espèces de la forêt littorale inféodées à ce milieu telles Manilkara obovata, Terminalia catapa, Callophyllum inophyllum, au profit des structures hôtelières (Angoni et al. 2013). En 2004, plus de 65 \% des hôtels étaient localisés à moins de $100 \mathrm{~m}$ de la côte. Il en résulte donc une dégradation intense de la forêt littorale du fait de l'occupation anarchique de la bande côtière (Angoni 2014). À cela s'ajoute le phénomène de l'érosion côtière à Kribi (Angoni et al. 2013).

5 Face à la dégradation progressive de cet écosystème, il devient urgent de mener une étude de la filière bois et identifier les espèces ciblées et évaluer les impacts. Certaines essences de mangrove (Rhizophora sp., Manilkara sp., Uapaca sp.) sont d'excellents combustibles, tandis que d'autres (Uapaca, Lophira alata) participent à la fabrication de divers matériels utilisés dans la vie quotidienne ainsi qu'à la construction des habitations.

C'est dans ce cadre que l'étude s'est fixée pour objectif principal d'identifier les différentes formes d'utilisation du bois dans les villages côtiers de Kribi.

7 De manière spécifique le travail vise à :

- identifier les espèces ligneuses utilisées dans les activités des populations côtières ;

- identifier les combustibles alternatifs utilisés ;

- identifier les sources d'approvisionnement.

\section{Méthodologie}

\section{Site d'étude}

8 Les villages concernés par l'étude se trouvent dans l'arrondissement de Kribi I (Nziou, Elabe, Ebouyé, Mpolongwé, Bebambwé, Londji I et II) et l'arrondissement de Campo (Campo Beach, Campo, Ebodjé, Lolabé, Nlende Dibé, Eboundja, Grand Batanga et Lobé). L'activité principale est la pêche par les hommes et le fumage du poisson par les femmes. 


\section{Méthode d'identification des types d'utilisation du bois}

9 Une enquête s'est faite dans les villages rencontrés le long de l'axe routier Kribi-Campo. Ainsi 8 villages sur 24 identifiés ont été retenus pour faire l'objet des enquêtes. Il s'agit de Campo Beach, Campo, Ebodjé, Lolabé, Nlende Dibé, Eboundja, Grand Batanga et Lobé. Au total donc, $25 \%$ des ménages recensés (recensement de ERE Développement 2001) (Angoni 2005) dans ces villages ont été interrogés. Ce qui faisait un échantillon de 149 ménages.

Les enquêtes ont été effectuées sur la base du questionnaire préalablement élaboré. Les entretiens se sont déroulés au hasard des rencontres avec des personnes disponibles. Les informations recueillies au moyen du questionnaire portaient sur les caractéristiques socio-professionnelles de la personne, les espèces végétales utilisées par les populations côtières dans leurs activités quotidiennes et les activités nécessitant l'utilisation du bois.

Figure 1 : Localisation des villages

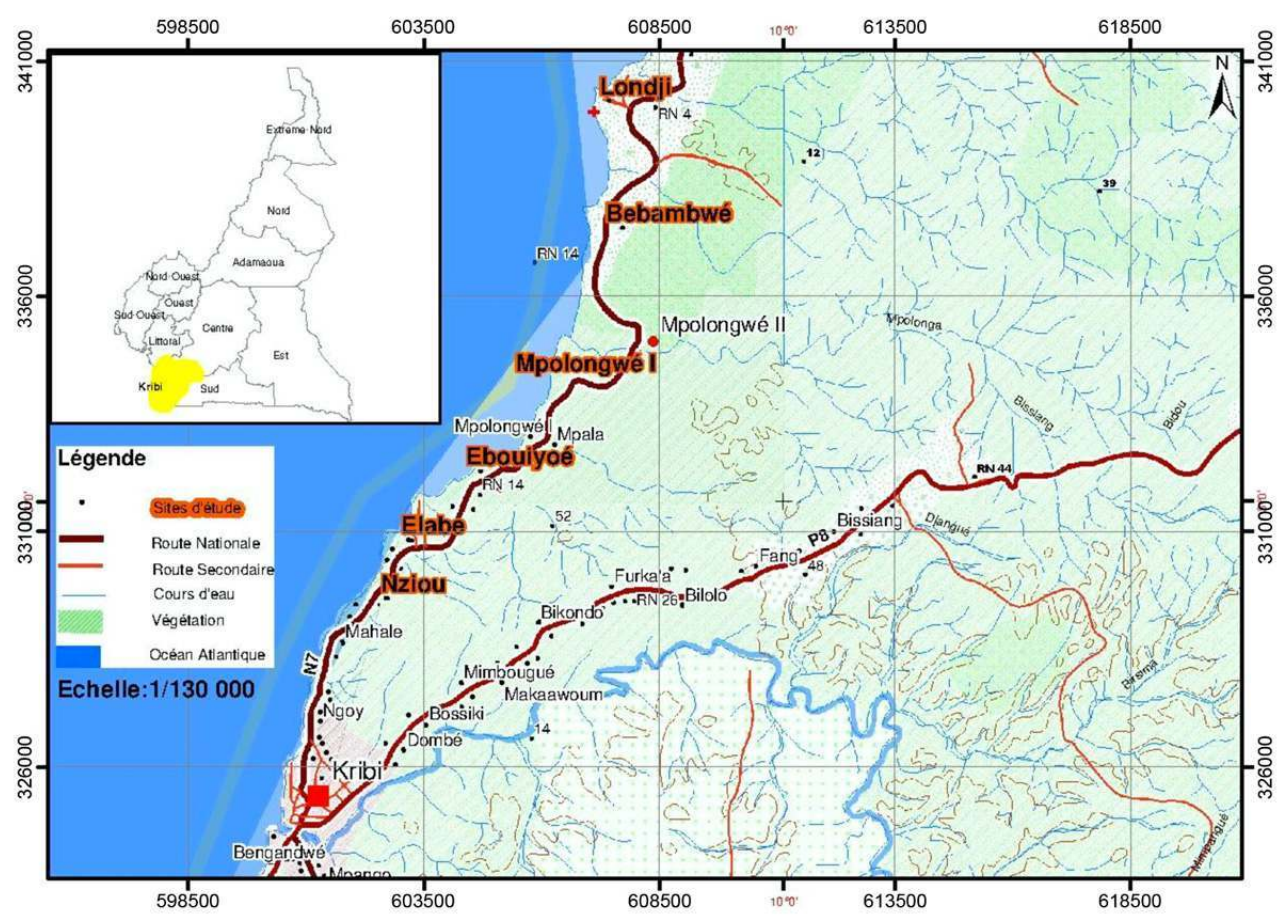

\section{Méthodologie d'étude de la filière bois de feu}

\section{Échantillonnage}

11 L'échantillonnage s'est effectué suivant la méthodologie Gold Standard utilisée par Chesnes (2009) ; d'après la procédure d'enquête, au minimum $10 \%$ des bénéficiaires du projet doivent être interrogés. Avec la recherche d'un échantillon représentatif d'une population de 500 fumeuses à une erreur de $10 \%$, la taille de l'échantillon est de 82 fumeuses (détail de calcul ci-dessous).

$\mathrm{n}_{\mathrm{o}}=\left(C V^{2} \cdot \mathrm{t}^{2} \alpha, \mathrm{v}\right) / \mathrm{e}^{2}$

$\mathrm{n}=\mathrm{n}_{\mathrm{o}} /\left(1+\mathrm{n}_{\mathrm{o}} / \mathrm{N}\right)$

En assumant $\mathrm{CV}=0,50$ pour la variable de consommation de bois de fumage dans la zone 
du projet, et un niveau de confiance $=0,95 . \mathrm{e}=$ erreur probable (on choisit $\mathrm{e}=10 \%$ ). $\mathrm{N}=$ taille de la population (500 fumeuses). $t \alpha,{ }_{\mathrm{V}}$ est la valeur de la loi statistique de Student pour un niveau de confiance de $95 \%$.

\section{Enquêtes auprès des fumeuses de poisson}

Des enquêtes ont été réalisées auprès des fumeuses de poisson ayant accès aujourd'hui à des fumoirs améliorés. Les informations recherchées concernent l'identification des espèces ligneuses utilisées comme bois de feu, les combustibles autres que le bois de feu, les différents lieux d'approvisionnement en bois de feu.

L'identification des espèces ligneuses se fait à partir des fiches de consommation de bois remises à chaque femme fumeuse de poisson. Les fumeuses doivent reporter sur ces fiches les dimensions (longueur et diamètre) de chaque morceau de bois utilisé lors du fumage ainsi que le nom vernaculaire ou courant du bois. Les appellations scientifiques de ces espèces seront déterminées à travers la recherche et la collecte des spécimens qui sont identifiés sur le terrain et confirmés à l'Herbier National.

\section{Données quantitatives}

Les données quantitatives concernent l'estimation de la consommation du bois par fumage. Un facteur de conversion de quantité de bois consommé en volume exprimé en $\mathrm{m}$ ${ }^{3}$ est appliqué. Chaque morceau de bois est mesuré (longueur et diamètre). Pour le calcul des volumes de bois, nous avons repris la formule utilisée par Feka et al. (2009) dans la réserve de Douala-Edéa. Cette formule assimile chaque morceau de bois à un cylindre de diamètre $\mathrm{D}$ et de longueur $\mathrm{L}$. Un facteur de correction de forme « $\mathrm{f}$ » est appliqué et dans le cas présent la valeur $\mathrm{f}=0,6$.

\section{Description des types de fumoirs}

\section{Fumoir amélioré}

Un bon fumoir doit résister à la chaleur d'où l'importance du choix des matériaux de construction. Un fumoir amélioré est fabriqué en briques pleines et les jointures doivent être en ciment et la partie supérieure devant supporter les claies de fumage, coulée en béton armé. C'est un fumoir qui a environ un mètre de haut, une longueur de deux mètres et une largeur d'un mètre. La partie supérieure est recouverte de grillage et un couvercle est fabriqué avec de la tôle. À la base du fumoir, une ouverture permet de disposer du bois pour y allumer du feu (Figure 2).

\section{Fumoir traditionnel}

16 Le fumoir traditionnel est composé d'un demi-fût ou autre objet recyclé auquel la partie supérieure est recouverte d'un grillage servant à fumer du poisson. Dans ce fumoir il n'existe pas de couvercle. À la base un orifice permet d'entretenir le feu de bois (Figure 3). 
Figure 2 : Fumoir amélioré

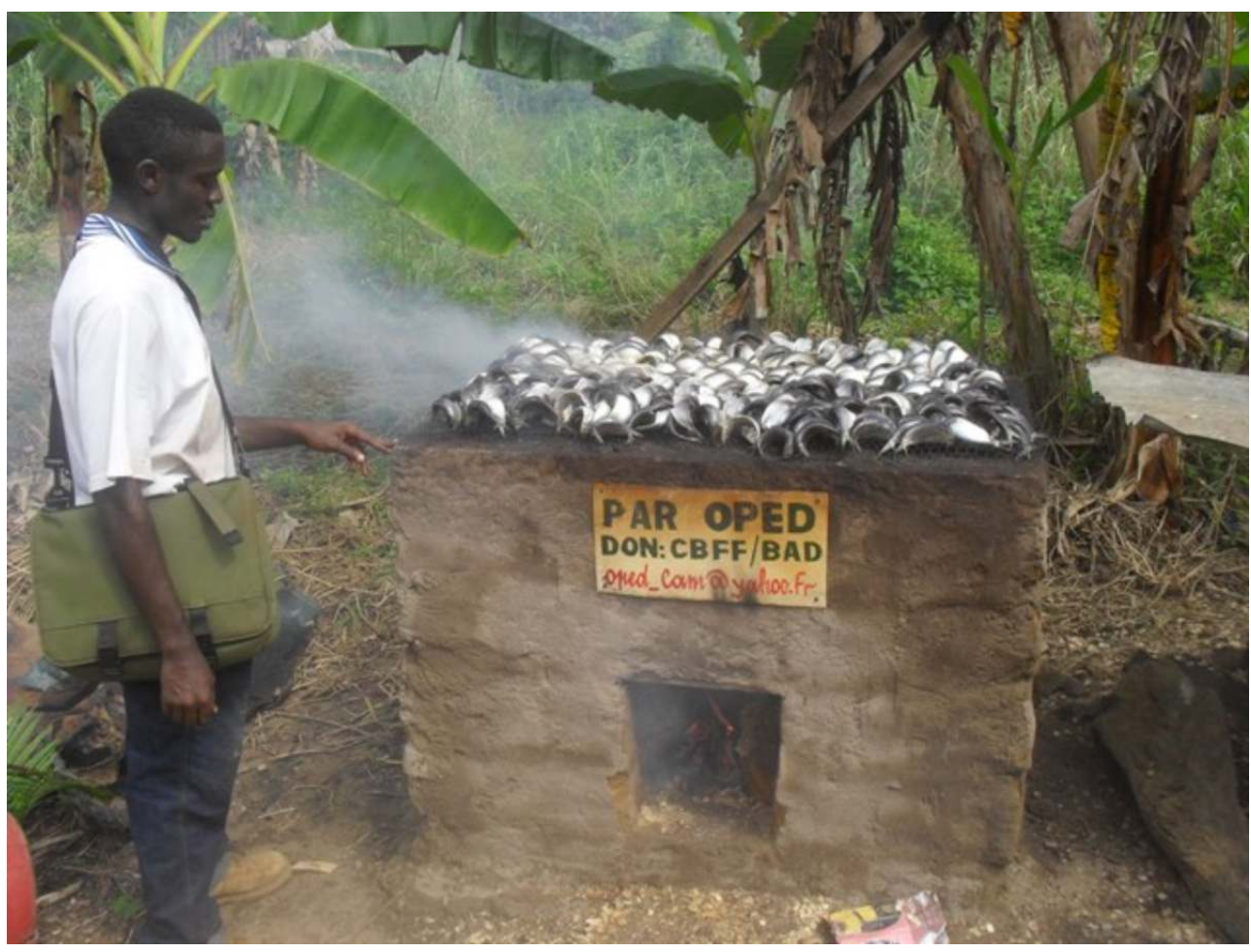

(c) A. Pouokam Tatchim

Figure 3 : Fumoir traditionnel

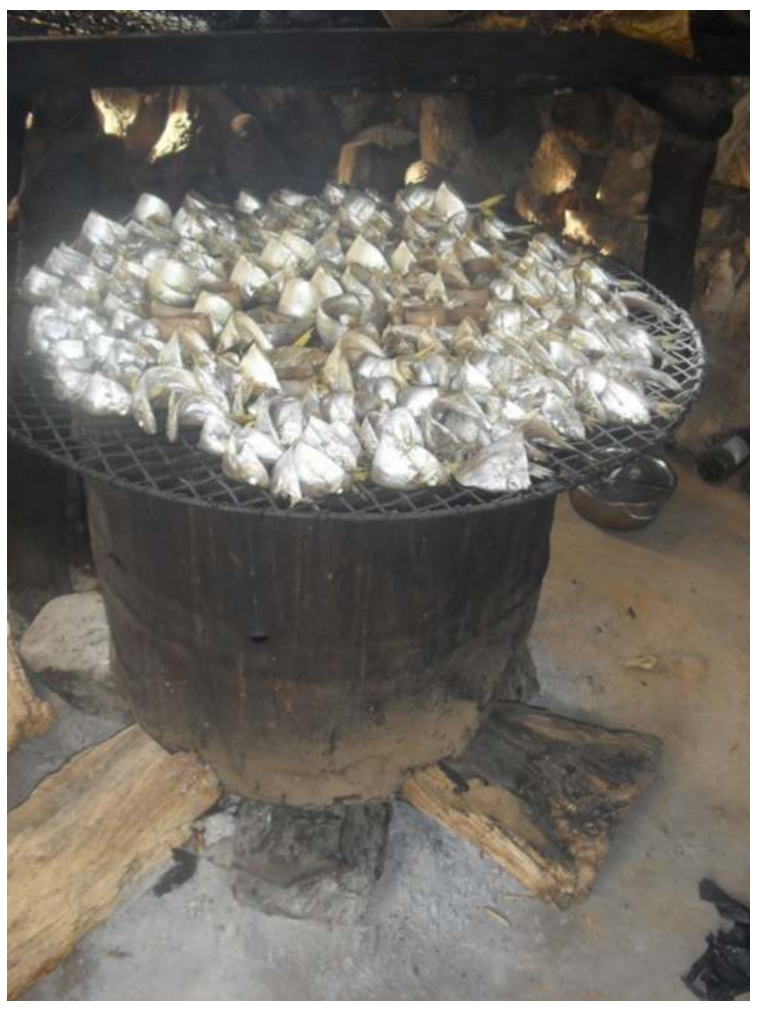

(c) A. Pouokam Tatchim 


\section{Résultats}

\section{Activités des populations}

Les enquêtes auprès des populations ont touché $72 \%$ d'hommes et $28 \%$ de femmes. En fonction des catégories socio-professionnelles, $63 \%$ des personnes interrogées sont des pêcheurs, $21 \%$ des agriculteurs, $5 \%$ des commerçants, $3 \%$ des fonctionnaires et $3 \%$ des maçons (Figure 4). La pêche reste la principale activité des populations en zone côtière et par conséquent la principale source de revenus dans ces villages. Toutes les autres activités des populations sont en rapport avec la pêche, il s'agit de la fabrication des pirogues, des rames, des filets et le fumage du poisson.

Figure 4 : Activités des populations locales

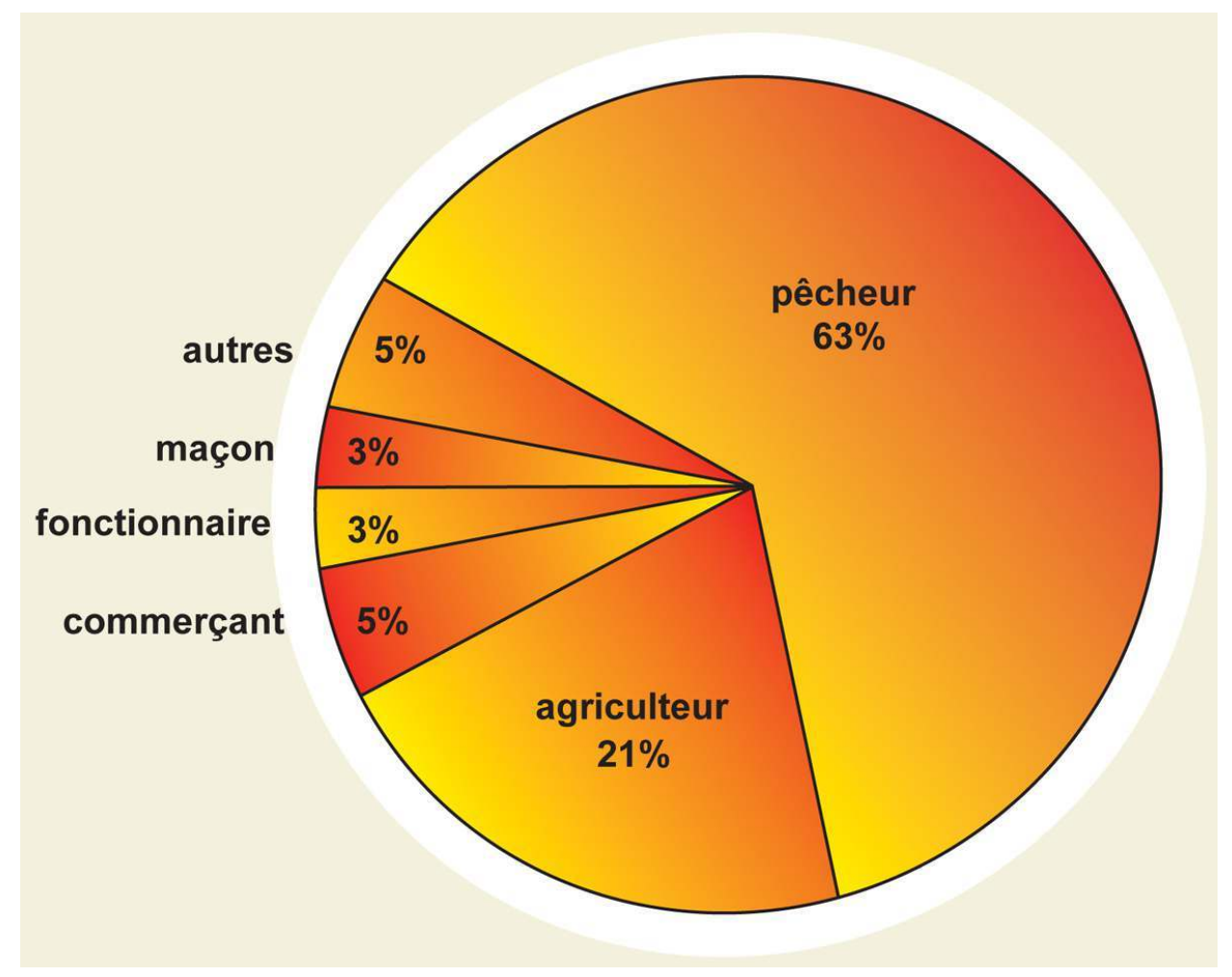

\section{Activités consommatrices de bois}

Cinq activités principales consomment du bois dans la zone côtière entre les villes de Kribi et de Campo: fabrication des pirogues, construction des maisons, bois de feu, fumage du poisson et médecine traditionnelle.

\section{Fabrication des pirogues et impact sur la biodiversité}

19 La pirogue à rame est le principal outil du pêcheur à faible revenu au Cameroun. Une pirogue se renouvelle tous les quatre ans; le diamètre utile est de $70 \mathrm{~cm}$ pour une longueur de 4 mètres. Ainsi 92 pirogues ont été recensées dans les sites Campo, Ebodjé, Lolabé et Eboundja (Figure 5). 
$\mathrm{Au}$ niveau de l'impact sur l'environnement, ce sont 77 arbres abattus pour la fabrication des pirogues et créant des ouvertures dans la canopée.

Figure 5 : Nombre de pirogues en fonction des ports de pêche

\begin{tabular}{|c|c|}
\hline Port de pêche & Nombre de pirogues \\
\hline Campo & 15 \\
\hline Ebodjé & 32 \\
\hline Lolabé & 27 \\
\hline Eboundja & 18 \\
\hline Total & 92 \\
\hline
\end{tabular}

\section{Essences végétales utilisées par les populations}

Il ressort de l'enquête effectuée que 14 espèces d'arbres sont utilisées pour les besoins de la vie quotidienne (Figure 6). Parmi celles-ci, on a identifié Azobé (Lophira alata), Assambi ( Uapaca sp.), Okoumé (Okoumea klaineana), Padouk (Pterocarpus soyauxii), Bibollo (Lovoa trichilioides) et Issati (Macaranga sp.) qui sont plus utilisées que les espèces telles que Bahia (Mitragyna ciliata), Bubinga (Guiboursia tessmannii), Ilomba (Pycnanthus angolensis), Iroko ( Milicia excelsa), Movingui (Distemonanthus benthamianus), Palétuvier (Rhizophora racemosa), Parasolier (Musanga cecropioides) et manguier (Mangifera indica).

Figure 6 : Espèces exploitées dans la zone côtière

\begin{tabular}{|c|c|c|c|}
\hline Famille & Nom scientifique & Nom commercial/Nom local & Utilisations \\
\hline Ochnaceae & Lophira alata Banks ex C.F.Gaertn. & azobé & \multirow{7}{*}{ Fabrication de pirogue, fumage du poisson } \\
\hline Burseraceae & Aucoumea klaineana Pierre & okoumé & \\
\hline Caesalpiniaceae & Distemonanthus benthamianus Baill. & movingui & \\
\hline Fabaceae & Pterocarpus soyauxii Taub. & padouk & \\
\hline Meliaceae & Lovoa trichilioides Harms & bibollo & \\
\hline Fabaceae & Guibourtia tessmannii (Harms) J.Léonard & bubinga & \\
\hline Fabaceae & Milicia excelsa (Welw.) C.C.Berg & iroko & \\
\hline Euphorbiaceae & Uapaca guineensis Müll.Arg. & assambi & \multirow{2}{*}{$\begin{array}{l}\text { Fabrication de pirogue, bois de chauffe, } \\
\text { construction de maisons }\end{array}$} \\
\hline Euphorbiaceae & Macaranga sp. & issati & \\
\hline Rubiaceae & Mitragyna ciliata Aubrév. et Pellegr. & bahia & Fabrication de pirogue, bois de chauffe \\
\hline Myristicaceae & Pycnanthus angolensis (Welw.) Warb. & ilomba & $\begin{array}{l}\text { Construction de maisons, fabrication de } \\
\text { pirogue }\end{array}$ \\
\hline Rhizophoraceae & Rhizophora racemosa G.Mey. & palétuvier & Bois de chauffe, fumage du poisson \\
\hline Cecropiaceae & Musanga cecropioides R.Br.apud Tedlie & parasolier & Construction de maisons, bois de chauffe \\
\hline Anacardiaceae & Mangifera indica L. & manguier & Bois de chauffe, fruits \\
\hline
\end{tabular}

Selon les populations, certaines espèces (Lophira alata), Assambi (Uapaca sp.), Okoumé ( Aucoumea klaineana), Padouk (Pterocarpus soyauxii), Bibollo (Lovoa trichilioides) et Issati ( Macaranga sp.) ainsi utilisées ont beaucoup diminué autour des habitations. En effet, il faut aujourd'hui aller plus loin dans la forêt pour les trouver. Cette raréfaction serait le fait de trois principaux facteurs : l'exploitation forestière (48\%), l'agriculture $(34 \%)$ et l'utilisation anarchique de ces essences par les populations (18\%).

\section{Fumage du poisson et habitudes culinaires}

La conservation du poisson n'est pas le seul objectif recherché dans le fumage de poisson. Certains mets traditionnels sont préparés avec du poisson fumé leur donnant une saveur et un goût particuliers. Aussi les fumeuses ont-elles le souci d'obtenir un produit de bonne qualité et donner une coloration et une bonne odeur au poisson fumé. Elles ont ainsi développé des préférences pour certaines espèces de bois pour leurs propriétés calorifiques et leur capacité à influencer les propriétés organoleptiques du poisson fumé. 
Les espèces de bois les plus recherchées sont le Sambi (Uapaca guineensis), 37 \% des fumeuses sont de cet avis, vient ensuite l'Azobé (Lophira alata) $25 \%$ et enfin le manguier ( Mangifera indica) $13 \%$ (Figure 7).

Figure 7 : Importance relative de préférence en bois de feu pour le fumage

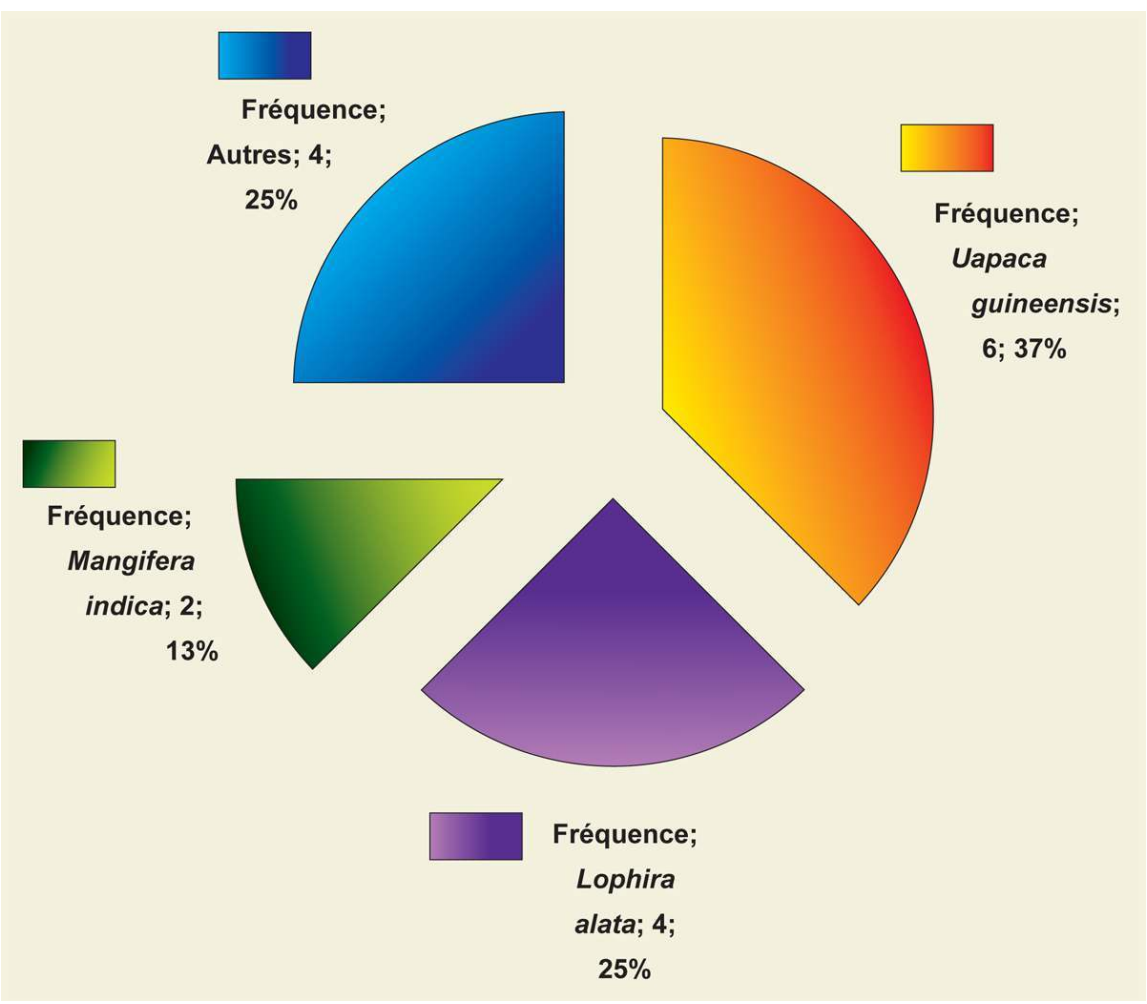

\section{Sources d'énergie alternatives}

Dans le souci de palier une absence de bois approprié au fumage du poisson, de nombreuses fumeuses ont développé l'usage d'autres sources d'énergie dites alternatives. Il s'agit de coque de noix de coco, déchets de cuisine (peau de plantain et de manioc) (Figure 8). Ces sources d'énergies sont prisées du fait de leurs capacités à améliorer les propriétés calorifiques du fumage. Elles ne se substituent pas au bois de feu, mais sont utilisées en complément. Ces sources d'énergie alternatives (Figure 8 ; Figure 9) jouent un rôle important dans la réduction de la consommation de bois de feu. Près de $60 \%$ de fumeuses ont adopté ces sources d'énergie.

Figure 8 : Quelques sources d'énergie alternatives au bois de feu

\begin{tabular}{l|l}
\hline Sources d'énergie alternatives & Observations \\
\hline Coque de noix de coco & $\begin{array}{l}\text { Gain de } 5 \text { à } 10 \% \text { d'énergie, apporte une coloration particulière et un } \\
\text { goût agréable au poisson fumé }\end{array}$ \\
\hline Déchets de cuisine (peau de banane plantain et de tubercule de manioc) & Gain de 5 à $10 \%$ d'énergie \\
\hline Sous-produit de l'industrie du bois (copeau et sciure) & $\begin{array}{l}\text { Gain de } 5 \text { à } 10 \% \text { d'énergie, donne un goût agréable et une bonne } \\
\text { coloration au poisson fumé }\end{array}$ \\
\hline Écailles de poisson & Donne une coloration dorée au poisson fumé \\
\hline Déchets végétaux (branches de palmier, écorces...) & Réduit la consommation de bois de 5 à $10 \%$ \\
\hline Coque de noix de palme & Permet au poisson fumé d'avoir une bonne coloration \\
\hline
\end{tabular}


Figure 9 : Sources d'énergie alternatives
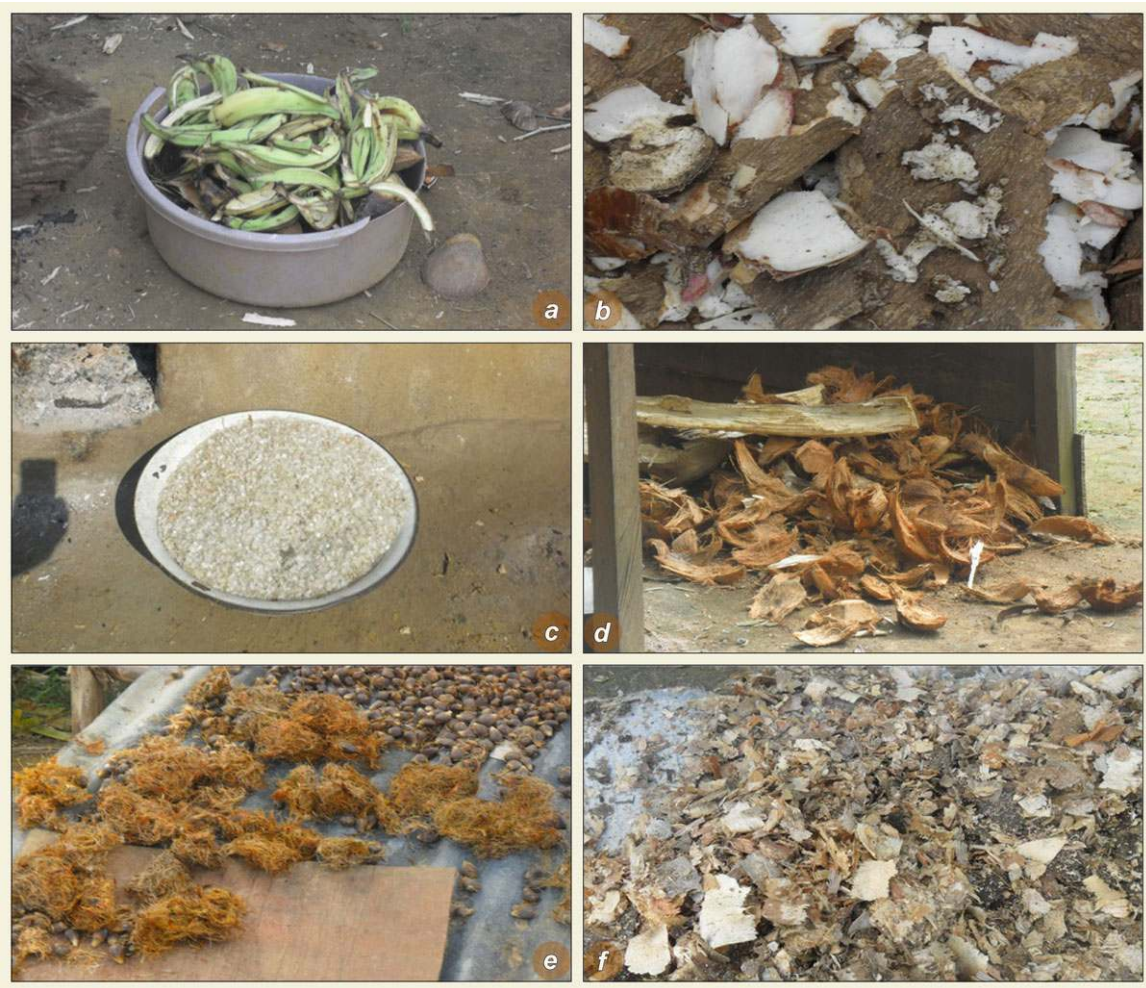

$a$ : Peaux de banane plantain ; $b$ : Peaux de manioc ; $c$ : Écailles de poisson ; $d$ : Coques de noix de coco ; e : Fibres de noix de palme ; $f$ : Copeaux de bois

(c) A. Pouokam Tatchim

\section{Circuit de commercialisation du bois de feu au Cameroun}

Concernant l'approvisionnement en bois de feu par l'intermédiaire des circuits commerciaux, les principaux fournisseurs sont les scieries, les menuiseries, les petits vendeurs de bois et les abatteurs. Les enquêtes montrent que $23 \%$ des fumeuses ont recours à l'achat du bois de feu. Les prix varient en fonction du chargement; pour un véhicule de cinq places plein les prix vont de 10000 FCFA à 15000 FCFA. C'est un marché régi par la demande des fumeuses et par la période de pêche (fructueuse ou pas). L'approvisionnement auprès des scieries et des menuiseries se fait pour les villages proches du centre de la ville de Kribi où sont localisées les scieries et les menuiseries. Les fumeuses se rendent dans ces usines de transformation du bois pour s'approvisionner en déchets de bois. Les prix sont en fonction de l'importance du chargement et vont de 2000 à 3000 FCFA le mètre cube. Le bois est aussi vendu en tas comprenant 5 à 10 morceaux. Le volume moyen du tas vendu est de $0,64 \mathrm{~m}^{3}$ pour un coût de 1000 FCFA.

\section{Efficacité des fumoirs améliorés par rapport au fumoir traditionnel}

La durée du fumage est passée de deux jours en fumoir traditionnel à une journée en fumoir amélioré pour une quantité moyenne de $30 \mathrm{~kg}$ de poissons. Cette durée est fonction de la quantité de poisson et surtout du type de poisson. La consommation moyenne de bois de feu en fumoir traditionnel après 10 essais est de $6,3 \mathrm{~m}^{3}$ contre $3,4 \mathrm{~m}^{3}$ 
en fumoir amélioré. Les tests énergétiques permettent ainsi d'observer des réductions de consommation de bois au-delà de $50 \%$ (Figure 10 ).

Figure 10 : Rendement énergétique des fumoirs améliorés

\begin{tabular}{|c|c|c|}
\hline & Gain énergétique & Observations \\
\hline Fumoirs traditionnels : claies et demi-fûts & $2,5 \mathrm{~kg}$ de bois pour $1 \mathrm{~kg}$ de poissons frais & $\begin{array}{l}\text { Les ouvertures des anciens fumoirs provoquent une } \\
\text { déperdition de la chaleur et par conséquent un usage } \\
\text { plus important du bois }\end{array}$ \\
\hline Fumoirs améliorés & $\begin{array}{l}\text { Gain de } 50 \% \text { soit } 1,25 \mathrm{~kg} \text { bois } / 1 \mathrm{~kg} \text { de } \\
\text { poissons frais }\end{array}$ & $\begin{array}{l}\text { Les quatre murs du fumoir amélioré permettent } \\
\text { une concentration de la chaleur et réduisent la } \\
\text { consommation du bois }\end{array}$ \\
\hline
\end{tabular}

\section{Impacts associés aux fumoirs}

\section{Impacts socio-économiques des fumoirs améliorés}

Sur le plan de la santé, il y a moins d'effort à fournir, moins d'exposition à la fumée et par conséquent aux maladies.

Le marché du poisson fumé dispose d'une meilleure qualité et valeur nutritive, de meilleures possibilités de commercialisation du produit, ce qui accroît les revenus des fumeuses.

Les fumeuses bénéficiant d'un fumoir amélioré utilisent moins de bois et gagnent du temps. Les avantages vont aussi directement dans la préservation de l'environnement grâce à la gestion des stocks de bois.

Au-delà du gain socio-économique immédiat pour les fumeuses de poisson, l'amélioration des fumoirs et l'usage des combustibles alternatifs permettent de réduire de façon durable la pression sur les formations végétales. $100 \%$ de femmes ayant bénéficié des fumoirs améliorés nous ont fait savoir que leur fréquence de collecte de bois s'est réduite de près de $50 \%$. La réduction du prélèvement devrait également réduire les problèmes liés à la régénération et au maintien des écosystèmes des mangroves. En de nombreuses zones, les prélèvements ont entraîné une ouverture trop brutale du couvert. Les fumoirs améliorés permettent de faibles émissions de fumées (riches en $\mathrm{CO}_{2}$ ) responsables de l'effet de serre. Les fumoirs améliorés constituent par conséquent également un moyen de lutte contre le changement climatique.

Figure 11 : Comparatif des fumoirs améliorés et des fumoirs traditionnels

\begin{tabular}{|c|c|c|c|c|c|c}
\hline$N^{\circ}$ & Nom local & Nom scientifique & Famille & $\begin{array}{c}\text { Temps de fumage } \\
\text { (ancien fumoir) }\end{array}$ & $\begin{array}{c}\text { Temps de fumage } \\
\text { (fumoir amélioré) }\end{array}$ & $\begin{array}{c}\text { Pourcentage de } \\
\text { temps récupéré }\end{array}$ \\
\hline 1 & Bonga & Ethmalosa fimbriata & Clupeidae & $72 \mathrm{~h}$ & $24 \mathrm{~h}$ & $63 \%$ \\
\hline 2 & Bilolo & Sardinella maderensis & Clupeidae & $36 \mathrm{~h}$ & $12 \mathrm{~h}$ & $63 \%$ \\
\hline 3 & Mio & & Clupeidae & $24 \mathrm{~h}$ & $6 \mathrm{~h}$ & $63 \%$ \\
\hline 4 & Bar & Pseudotolithus typus & Sciaenidae & $72 \mathrm{~h}$ & $36 \mathrm{~h}$ & $50 \%$ \\
\hline 5 & Raie & Manta birostris & Myliobatidae & $88 \mathrm{~h}$ & $48 \mathrm{~h}$ & $50 \%$ \\
\hline
\end{tabular}

\section{Discussions}

Cette étude a permis de constater que les espèces les plus prisées pour le fumage du poisson sont le Sambi (Uapaca guineensis) $37 \%$ et l'Azobé (Lophira alata) $25 \%$. D'après Knockaert (1999), la coloration du poisson varie avec les types de bois. Nfotabong Atheull (2011) qui a étudié les impacts des activités anthropiques sur la structure des forêts de 
mangrove à Kribi, a montré qu'en dépit de l'usage des palétuviers Rhizophora sp. comme bois de chauffe, les populations collectent l'Azobé (Lophira alata) qui présente les mêmes propriétés calorifiques que le palétuvier rouge (Rhizophora racemosa).

Le scénario concernant l'usage du bois de mangrove comme combustible pour le fumage observé dans les mangroves de Kribi diffère de celui des autres zones de mangrove du Cameroun où le bois de mangrove est la principale ressource utilisée dans le fumage du poisson. Notre étude montre que le bois de mangrove ne représente à Kribi que $5 \%$ des espèces ligneuses utilisées comme combustible. Selon Ajonina et Usongo (2001), l'espèce Rhizophora est prisée pour son pouvoir calorifique et sa capacité à se consumer lentement, sans séchage préalable. Feka et Manzano (2008) affirment que le bois de mangrove est utilisé à $62 \%$ pour le fumage dans les mangroves situées dans le sud-ouest Cameroun. Une étude menée dans les mangroves de l'estuaire du Cameroun et la zone de Bakassi Njifonjou (2009) (Ajonina 2008) a trouvé que la consommation de bois de mangrove était respectivement de $47 \%$ et $50 \%$ pour le fumage. Ajonina (2001) dans une étude dans la réserve de faune de Douala-Edéa rejoint cette tendance en affirmant que le fumage dépend presque entièrement $d u$ bois de mangrove. Par contre, les résultats de notre étude montrent une faible consommation du bois de mangrove pour le fumage; ceci pourrait s'expliquer d'une part par la présence de plusieurs formations naturelles dans la zone, d'autre part par la faible surface occupée par cette mangrove relativement aux autres formations. Nfotabong Atheull (2011) conforte ces résultats; selon lui, la mangrove de Nziou par exemple a fortement été endommagée par l'installation des habitations modernes au bord de la mer.

Les processus d'érosion côtière marquent les plages du Golfe de Guinée. Le rythme d'évolution actuelle des côtes sableuses dans le Golfe de Guinée entre la Côte-d'Ivoire et le Cameroun a suscité des études d'application. Le recul du trait de côte est très significatif avec des vitesses de l'ordre de 1 à 5 , voire 10 mètres par an.

34 À l'échelle européenne, le processus d'érosion côtière concerne $20 \%$ du linéaire côtier, équivalant en superficie à une perte annuelle de $15 \mathrm{~km}^{2}$. En France, un quart des côtes est concerné soit un linéaire de $1720 \mathrm{~km}$ dont les deux tiers de zones sableuses.

Cette étude a permis de dégager les performances des fumoirs améliorés quant à la consommation d'énergie et au gain de temps. Feka et Manzano (2008) cités par Chesnes (2009) ont également travaillé dans la mise en œuvre d'un projet de fumoirs améliorés pour le fumage du poisson. Des réductions de consommation de $30 \%$ et des gains de temps de 4 jours en fumoir traditionnel à 2 jours en fumoir amélioré pour des espèces de poisson comme le Bonga (Ethmalosa fimbriata) et de $24 \mathrm{~h}$ en fumoir traditionnel à $8 \mathrm{~h}$ en fumoir amélioré, pour le fumage des sardines et aussi d'autres espèces.

\section{Conclusion}

Cette étude a porté sur l'évaluation de l'utilisation du bois de feu dans les pêcheries côtières de Kribi. Elle a permis d'identifier 13 espèces ligneuses couramment utilisées dans le fumage de poisson. On note l'utilisation d'autres combustibles que le bois de feu, il s'agit notamment des déchets issus de l'industrie du bois (sciure, copeaux, écorces...), des déchets végétaux et animaux qui contribuent de façon significative à la réduction de la consommation du bois de feu. 

passée à $1,25 \mathrm{~kg}$ de bois pour $1 \mathrm{~kg}$ de poisson frais, soit une réduction de $50 \%$. Les risques de maladies liées à l'émission des fumées et à l'exposition à la chaleur ont nettement baissé, le temps de fumage est réduit avec les fumoirs améliorés pour toutes les espèces de poissons identifiées. Cette technologie permet de lutter contre le réchauffement climatique à deux niveaux, d'une part elle contribue à la réduction de la déforestation, d'autre part elle permet une faible émission de fumée porteuse de gaz à effet de serre.

Le fumage de poisson dans les mangroves de Kribi présente un visage différent de celui observé dans les autres zones de mangrove du Cameroun, concernant l'usage de ce bois comme combustible à cause de l'absence des espèces Rhizophora sp. Le bois de mangrove ( Terminalia catapa, Manilkara obovata et Rhizophora sp.) est très faiblement utilisé dans le fumage; pourtant ses vertus calorifiques et sa capacité à influencer les propriétés organoleptiques sont bien connues des populations. En conséquence, on utilise deux espèces très prisées de forêt, Sambi (Uapaca guineensis) et Azobé (Lophira alata) qui malheureusement se raréfient.

\section{BIBLIOGRAPHIE}

Ajonina G. N. 2001 - Preliminary quantitative impact assessment of wood extraction on the mangrove of Douala-Edea forest reserve, Cameroon. Tropical biodiversity 7 (2-3) : 137-149.

Ajonina G. N. 2008 - Inventory and modeling mangrove forest stand dynamics following different levels of wood exploitation pressures in the Doula-Edea Atlantic coast of Cameroon, Central Africa. Thèse, Albert-Ludwigs-Universtät, Freiburg im Breisgau, 215 p.

Ajonina G. N. \& Usongo L. 2001 - Preliminary assessment of wood utilization impact and conservation prospects of coastal mangrove forest of Douala-Edea reserve Cameroon. Tropical biodiversity 7 (2-3) : 137-149.

Angoni H. 2005 - Biologie et écologie des tortues marines en rapport avec les écosystèmes côtiers. Conservation et aménagement. Thèse de Doctorat de troisième Cycle. Université de Yaoundé I, Cameroun. 93 p.

Angoni H. 2014 - Les tortues marines du Cameroun et les milieux côtiers. Presses Académiques Francophones, $116 \mathrm{p}$.

Angoni H., Ngodo Melingui J. B., Kono L., Amougou Akoa \& Fretey J. 2013 - Étude de la végétation des côtes atlantiques à la périphérie du parc national de Campo Ma'an au Sud du Cameroun. Annales de la Faculté des Sciences, 12 p.

Chesnes M. 2009 - Renouvelabilité de la ressource en bois de mangrove vis-à-vis d'usages dans la réserve de Douala-Edéa, Cameroun : préparation à la mise en place d'un projet MDP pour l'amélioration de fumoirs à poissons. Mémoire de stage. AgroParisTech-ENGREF, centre de Montpellier, 86 p.

FAO 2006 - Document de politique et stratégie pour la gestion durable des écosystèmes de mangroves du Cameroun. FAO, Yaoundé, Cameroun, 37 p.

Revue d'ethnoécologie, 7 | 2015 
Feka N. Z., Chuyong G. B. \& Ajonina G. N. 2009 - Sustainable Utilization of mangrove using improved fish smoking systems: a management perspective from the Douala-Edea Wildlife reserve Cameroon, $85 \mathrm{p}$.

Feka N. Z. \& Manzano M. G. 2008 - The implications of wood exploitation for fish smoking on mangrove ecosystem conservation in the South West Province, Cameroon. Tropical Conservation Science 1 : 222-241.

Knockaert C. 1999 - Le fumage de poisson. Collection valorisation des produits de la mer. Brest, Ifremer, $174 \mathrm{p}$.

Mbog D. M. 2005 - TCP/CMR/2908 (A) Gestion participative et conservation de la diversité biologique des mangroves. FAO, $28 \mathrm{p}$.

Nfotabong Atheull A. 2011 - Impact of anthropogenic activities on the vegetation structure of mangrove forests in Kribi, the Nyong river mouth and Cameroon Estuary. Ph.D Thesis, Université Libre de Bruxelles-ULB, Brussels, Belgium / The University of Douala, Cameroon. 196 p.

\section{RÉSUMÉS}

Kribi est située sur la côte maritime du Cameroun, dans la région du Sud, chef-lieu du département de l'Océan. La bordure côtière Sud Cameroun est caractérisée par une végétation de mangroves qui fait partie du grand ensemble des mangroves du Rio Ntem. La pêche constitue la principale activité génératrice de revenus pour les populations. Pour la conservation et la transformation du poisson, le fumage y est largement pratiqué. Cette technique consomme d'énormes quantités de bois dont l'utilisation abondante pour le fumage de poisson constitue une menace pour les mangroves et la biodiversité en général. Ainsi cette étude qui s'étend de Kribi à Campo a pour objectif d'étudier les usages du bois et identifier les impacts de son exploitation dans l'environnement.

Des enquêtes auprès des communautés des pêcheurs, des acteurs de la filière fumage de poisson dont les fumeuses et les vendeurs de bois, des mesures biométriques ont été réunies au sujet des bois récoltés.

Il ressort de cette étude qu'il existe deux modes d'approvisionnement de bois. Les populations coupent directement le bois dans la forêt (auto-approvisionnement) ce qui s'explique par la proximité des forêts.

Le fumage représente $60 \%$ des différents usages du bois de feu. En comparaison avec le fumoir classique, les fumoirs améliorés permettent des réductions de la consommation de bois de l'ordre de $50 \%$. La consommation moyenne en fumoir traditionnel est de $6,8 \mathrm{~m}^{3}$ contre $3,4 \mathrm{~m}^{3}$ pour les fumoirs améliorés. Le bois de mangrove est de moins en moins utilisé par les fumeuses. Par contre, le Sambi (Uapaca guineensis) est une essence très prisée dans le fumage de poisson.

Kribi is situated in the Cameroon coastal zone, in the Southern Region and in the Ocean administrative department.

The coastal border in the South Cameroon is characterized by a mangrove vegetation which is part of the Rio Ntem mangrove zone.

Fishing activities constitutes the main income generating activity for the populations. For the conservation and the transformation of the fish, drying is widely practiced in the region. This technique consumes enormous quantities of wood causing severe threats on biodiversity.

This study which extends from Kribi to Campo has as objective, to study the uses of wood and to identify the impacts of its exploitation in the environment.

Inquiries with the fishermen communities, the actors of the fish drying activities in which the 
dryers and the salesmen of wood, the biometric measures were collected on harvested wood. It comes out of this study that there are two types of wood supply. The populations cut the wood directly from the forest because of the nearness of forests. Fish drying represents $60 \%$ of the various uses of the fire wood. In comparison with the classic drying method, the improved new drying system allows the reductions of the wood consumption in the level of $50 \%$. Average consumption in traditional drying system is about $6,8 \mathrm{~m} 3$ against $3,4 \mathrm{~m} 3$ for the new improved drying method. The wood of mangrove is more or less used by the dryers. On the other hand, Sambi (Uapaca guineensis) is most appreciated species in the fish drying activity.

INDEX

Mots-clés : fumage de poisson, fumoir amélioré, bois de feu, dégradation des forêts, zone côtière Keywords : fish drying, improved drying system, firewood, degradation of coastal forest zone Index géographique : Cameroun

\section{AUTEURS}

\section{HYACINTHE ANGONI}

Université de Yaoundé I, Faculté des Sciences, Département de Biologie et Physiologie Végétales, Cameroun

\section{ALPHONSE POUOKAM TATCHIM}

Université de Yaoundé I, Faculté des Sciences, Département de Biologie et Physiologie Végétales, Cameroun

\section{BERNARD ALOYS NKONMENECK}

Université de Yaoundé I, Faculté des Sciences, Département de Biologie et Physiologie Végétales, Cameroun

\section{ELIE NGUEKAM}

Organisation Pour l'Environnement et le Développement Durable (OPED), Kribi, Cameroun 